\title{
Die operative Therapie des Endometriumkarzinoms
}

\author{
Bernhard Krämer, Florin-Andrei Taran, Sara Y. Brucker
}

\begin{tabular}{|c|c|c|c|}
\hline \multicolumn{4}{|l|}{ Übersicht } \\
\hline Epidemiologie & 35 & Omentektomie & 41 \\
\hline Risikofaktoren & 35 & Therapie in den fort- & \\
\hline Symptome und Diagnostik & 36 & geschrittenen Stadien II-IV & 41 \\
\hline Operative Therapie & 37 & Therapie von Rezidiv & \\
\hline $\begin{array}{l}\text { Strategie der Lymphonodektomie } \\
\text { beim Endometriumkarzinom }\end{array}$ & 38 & und Metastasen & 42 \\
\hline
\end{tabular}

\section{Epidemiologie}

Das Endometriumkarzinom ist der häufigste maligne gynäkologische Tumor der Frau und steht nach dem Mamma-, Lungen- und dem kolorektalen Karzinom insgesamt an 4. Stelle. Typischerweise erkranken postmenopausale Frauen, aber in ca. 20\% der Fälle muss auch prämenopausal mit einer malignen Neoplasie gerechnet werden. Das mittlere Erkrankungsalter liegt aktuell bei etwa 69 Jahren, die Inzidenz steigt mit zunehmendem Lebensalter an. Die altersstandardisierte Mortalität hat in den letzten 30 Jahren abgenommen und geht weiterhin zurück, im internationalen Vergleich zeigt sich in Deutschland eine relativ niedrige Mortalität, was aber nicht darüber hinwegtäuschen darf, dass die krankheitsspezifische 5-Jahres-Mortalität noch bei $19 \%$ liegt.

\section{Risikofaktoren}

\section{Typ-I-Karzinom}

Vor allem für das sog. Typ-I-Karzinom (meistens endometrioides Adenokarzinom, östrogenabhängig, ca. 75-90\% der Fälle), welches die Mehrzahl der Korpuskarzinome ausmacht, spielen patientenbezogene Risikofaktoren eine erhebliche Rolle, die die Östrogenstimulation beeinflussen. Hierzu gehören:
- Übergewicht

- Diabetes

- Alter

- Nulliparität

- Östrogenüberschuss (z. B. östrogenproduzierende Tumore, alleinige Östrogentherapie)

- chronische Anovulation/Subfertilität (z. B. PCO-Syndrom): das Fehlen von Progesteron in der 2. Zyklushälfte führt zur Dauerstimulation der endometrialen Schleimhaut

- späte Menopause

Die Risikofaktoren Übergewicht und Diabetes führen beide zu einer schlechteren Prognose.

Oft geht den Typ-I-Karzinomen eine Endometriumhyperplasie voraus.

\section{Typ-II-Karzinom}

Die genauen Risikofaktoren für die Typ-II-Karzinome (v.a. serös, klarzellig, papillär oder adenosquamös, östrogenunabhängig, 10-20\% der Fälle) sind weniger bekannt. Sie entstehen meist in atrophem Endometrium aus einem intraepithelialen Karzinom, welches als Vorläuferläsion zu sehen ist. Typ-II-Karzinome sind weniger gut differenziert und haben eine schlechtere Prognose als Typ-I-Malignome. 
In neueren Untersuchungen zeigt sich aber, dass sich sowohl beim Typ-I- als auch beim Typ-II-Karzinom ätiologische Faktoren überschneiden. Letzteres scheint auch nicht so unabhängig vom Östrogeneinfluss zu sein, wie man zunächst vermutet hatte [1]. Innerhalb der Typ-II-Gruppe scheinen zusätzliche Heterogenitäten vorhanden zu sein [2]. Unklar ist gegenwärtig noch, warum bei einer ähnlichen Exposition gegenüber Risikofaktoren der eine oder der andere Typ entstehen kann.

\section{Fazit für die Praxis}

Es gibt 2 Typen des Endometriumkarzinoms. Die Aggressivität ist unterschiedlich, die bisher deutlich hervorgehobenen Unterschiede hinsichtlich der Ätiologie scheinen sich aber zu überlappen.

\section{Genetische Faktoren}

\section{Lynch-Syndrom}

DNA-Instabilität. Ein bekannter genetischer Risikofaktor ist das sog. Lynch-Syndrom (Syn.: hereditäres nicht polypöses Kolonkarzinom-Syndrom, HNPCC). Es handelt sich hierbei um eine seltene autosomal-dominant vererbte Keimbahnmutation in einem der für die Genomintegrität wichtigen DNA-Mismatch-Reparaturgene, was zur genomischen Instabilität führt (typisch für das Lynch-Syndrom ist die sog. Mikrosatelliteninstabilität) und in der Folge das Lebenszeitrisiko für Malignomerkrankungen erhöht.

Früher Erkrankungsbeginn. Neben dem gesteigerten Risiko für frühe kolorektale Karzinome erkranken Mutationsträgerinnen gehäuft am Endometrium- und Ovarialkarzinom, v. a. im jüngeren Lebensalter. Für das Endometriumkarzinom beträgt hierbei das mittlere Erkrankungsalter etwa 45 Jahre, außerdem finden sich öfters metachrone und synchrone Zweitneoplasien. Meistens wird ein endometrioides Endometriumkarzinom diagnostiziert.

Das Lynch-Syndrom ist ein bekannter Risikofaktor für bestimmte Karzinome im mittleren Lebensalter.

Anamnese und Genanalyse. Zur Abschätzung des Vorliegens eines Lynch-Syndroms muss eine exakte Eigenund Familienanamnese (gehäufte familiäre Tumorerkrankungen, Auftreten in jüngerem Lebensalter?) erfolgen. Die sog. Amsterdam-Kriterien sowie die Bethesda- und die SGO-Kriterien (Society-of-Gyneco-
logic-Oncology-Kriterien) helfen bei der weiteren Eingrenzung (s. Kasten). Bei der Testung von Tumormaterial bereits erkrankter Patientinnen können die Mikrosatelliteninstabilitäten im Tumorgewebe nachgewiesen werden, daraus ergibt sich ein etwa 25\%iges Risiko, dass tatsächlich ein Lynch-Syndrom bei einer weiteren genetischen Analyse durch Mutationsnachweis diagnostiziert wird.

Frühes Karzinomscreening. Praktisch bedeutet ein nachgewiesenes Lynch-Syndrom eine Screeningempfehlung auf ein Endometriumkarzinom ab dem 30. -35. Lebensjahr. Diese besteht aus einer jährlichen Transvaginalsonografie und einer ambulanten Endometriumbiopsie. Bei abgeschlossener Familienplanung sollte großzügig die Hysterektomie mit Adnexektomie beidseits empfohlen werden.

\section{Amsterdam-Kriterien}

- mindestens 3 Verwandte mit Kolonkarzinom oder einem HNPCC-assoziierten Karzinom, wobei eine Patientin davon erstgradig verwandt mit den beiden anderen sein muss und eine familiäre adenomatöse Polypose (FAP) ausgeschlossen sein muss

- mindestens 2 Generationen betroffen

- Diagnose bei mind. einem Patienten < 50. Lebensjahr

\section{BRCA-Mutation}

Die Assoziation zwischen Keimbahnmutationen in BRCA-Genen und dem Risiko für das Endometriumkarzinom ist noch kontrovers [3]. Als praktische Konsequenz einer möglichen Risikoerhöhung bei BRCA-1Mutationsträgerinnen erwägen die Studienautoren die präventive Hysterektomie, wenn ohnehin bei den Patientinnen die prophylaktische Adnexektomie durchgeführt wird [4].

\section{Symptome und Diagnostik}

\section{Symptome}

Das Kardinalsymptom des Endometriumkarzinoms ist die Blutung:

- postmenopausal

- prä- oder perimenopausale Metro-/Menorrhagie 


\section{Tipp für die Praxis}

Jede postmenopausale Blutung und die atypische Blutung in der Perimenopause muss histologisch abgeklärt werden. Dabei ist die sonografisch gemessene Endometriumdicke unerheblich.

Es ist bekannt, dass unter Tamoxifen-Einnahme das relative Risiko für die Entwicklung eines Endometriumkarzinoms 2- bis 3-fach höher ist und dass sich unter der Medikation sonografische Veränderungen am Endometrium zeigen können, die hauptsächlich in einer Verdickung der Schleimhaut imponieren [6]. Diese alleine rechtfertigen aber keine histologische Abklärung, sondern erst das Vorliegen einer Blutung.

Bei ca. $10 \%$ der Frauen mit postmenopausaler

Blutung zeigt sich ein Endometriumkarzinom [5].

Abdominalsymptome wie Schmerz, Blähungen, Völlegefühl oder Störungen der Blasen- und Darmfunktion sprechen bereits für eine fortgeschrittene Erkrankung.

\section{Diagnostik}

Ein Routinescreening bei asymptomatischen Frauen ohne Risikofaktoren wird nicht empfohlen, die Ausnahme bildet das bereits o.g. Lynch-Syndrom. Es hat sich auch keine Verminderung der Mortalität beim Screening von Hochrisikogruppen (Adipositas, Endometriumhyperplasie) gezeigt. Bislang gilt als praktische Empfehlung die Abklärung einer Endometriumdicke von $>5 \mathrm{~mm}$ in der Postmenopause.

Bildgebende Maßnahmen alleine sind nicht zielführend, sodass zur Diagnosesicherung von Vorstufen oder eines bereits invasiven Karzinoms die histologische Sicherung durch Hysteroskopie und fraktionierte Abrasio erfolgen soll.

Die durch Hysteroskopie gesteuerte und gezielte fraktionierte Abrasio ist der Goldstandard bei der Diagnostik des Endometriumkarzinoms.

\section{Endometriumhyperplasie als Vorstufe}

Wie bereits oben erwähnt sind Endometriumhyperplasien als Vorläufer des hormonabhängigen Karzinoms (Typ I) zu sehen. Sie werden durch die Abrasio diagnostiziert, anschließend gibt es folgende therapeutische Optionen:
- Hyperplasie ohne Atypie. Prinzipiell ist ein konservatives Vorgehen möglich, bei prämenopausalen Patientinnen wird eine Gestagentherapie mit hysteroskopischer Kontrolle und erneuter Abrasio empfohlen, bei postmenopausaler Situation ist großzügig die Hysterektomie zu erwägen, da auch in diesen Fällen mit einem Karzinomrisiko von 1-3\% gerechnet werden muss.

- Hyperplasie mit Atypie. Das Entartungsrisiko beträgt bis zu 30\% und ist damit als hoch anzusehen. Konsequenterweise ist sowohl prä- als auch postmenopausal die Hysterektomie zu empfehlen, bei Kinderwunsch ist nach Risikoabschätzung die konservative Therapie mit Gestagen und engmaschiger (alle 3 Monate) Re-Hysteroskopie/Abrasio vertretbar.

\section{Operative Therapie}

Es besteht Konsens, dass es sich beim Endometriumkarzinom um eine Tumorentität handelt, bei der das operative Staging prognostische Parameter liefert, die für eine weitere individualisierte Therapie herangezogen werden.

\section{Präoperative Vorbereitung bei nachgewiesenem Endometriumkarzinom}

Nicht selten zeigen sich bei den Patientinnen Komorbiditäten wie Adipositas, Hypertonie und Diabetes, die in die OP-Vorbereitung und zur Abschätzung des anästhesiologischen Risikos einbezogen werden müssen. Daneben gelten folgende Untersuchungen als Routine:

- Oberbauchsonografie (Lebermetastasen?)

- Labor

- Röntgenthorax (Lungenmetastasen?)

- Nierensonografie (Ausschluss Harnstau)

Zusätzliche Bildgebung durch CT oder MRT sollte nur speziellen Fragestellungen vorenthalten sein oder beim Vorliegen/Verdacht eines ausgedehnten Tumorwachstums mit Infiltration der Umgebung zum Einsatz kommen. In diesen Fällen ist ggf. auch die Narkoseuntersuchung mit Zystoskopie und Rektoskopie zur Einschätzung sinnvoll, falls sich entsprechende Hinweise nicht bereits bei der Hysteroskopie/fraktionierten Abrasio ergeben haben (siehe auch unten zur Therapie fortgeschrittener Karzinome). 


\section{Standardtherapie}

Grundsätzlich besteht die operative Standardtherapie nach histologischer Sicherung der Diagnose aus der Hysterektomie, der bilateralen Adnexektomie und der Entnahme einer Peritonealspülzytologie.

Standardtherapie sind Hysterektomie, bilaterale Adnexektomie und Entnahme einer Peritonealspülzytologie.

\section{Laparoskopie versus Laparotomie und vaginale Hysterektomie}

In den letzten Jahren haben sich die Möglichkeiten für das laparoskopische Vorgehen stark verbessert. Die schnellere Rekonvaleszenz mit meist kürzerer Hospitalisation im Vergleich zur Laparotomie und die assoziierte Verbesserung der kurz- und langfristigen Morbidität sprechen klar für die minimalinvasive Methode, welche in vielen Zentren als Standard etabliert ist. Die onkologische Gleichwertigkeit konnte für die frühen Stadien des Endometriumkarzinoms belegt werden [7]; für die fortgeschrittene Erkrankung und die HighRisk-Fälle (s.u.) ist die Datenlage hierzu noch nicht sicher ausreichend. Es muss auch darauf hingewiesen werden, dass unter Umständen die Laparoskopie aufgrund der Komorbiditäten und der Adipositas mancher Patientinnen schwierig sein kann und daher in einem Zentrum durchgeführt werden sollte, welches die Erfahrung und das Setting hinsichtlich Lagerung, Anästhesie und ggf. Verfügbarkeit der Schnellschnittdiagnostik anbieten kann.

Die vaginale Hysterektomie für das (auch frühe) Endometriumkarzinom sollte nicht als Standard angesehen werden.

Selektionierte Patientinnen mit einem hohen OP-Risiko (Alter, BMI, zahlreiche relevante Komorbiditäten) für das offene oder laparoskopische Staging, welches den potenziellen Nutzen dieser Zugänge überwiegt, können aber davon profitieren, wenn die Alternative ein Verzicht auf die Operation wäre. Studien zu frühen Endometriumkarzinomen bei operativen Hochrisikopatientinnen berichten über ähnliche Überlebensraten nach vaginaler Hysterektomie im Vergleich zum abdominalen Zugang [8,9].

\section{Ergänzende Therapie}

Ob die operative Standardtherapie weiter ergänzt oder modifiziert wird, hängt von folgenden Kriterien ab:

- Histologietyp

- Grading

- Eindringtiefe ins Myometrium

- Befall des Zervixstromas

\section{Ergänzende Therapieformen}

- Lymphonodektomie (LNE)

- Omentektomie

- Peritonealbiopsien

\section{Strategie der Lymphonodektomie beim Endometriumkarzinom}

Die Rationale für die Lymphonodektomie (LNE) leitet sich vom Lymphabfluss des Tumors im Bereich des Corpus uteri ab: Dieser vollzieht sich einerseits über die pelvinen Lymphknoten (LK), aber auch über Lymphbahnen, welche über die Adnexen direkt in die paraaortale Region drainieren können, weswegen paraaortale Lymphknoten auch bei freien pelvinen Lymphknoten positiv sein können [10,11]. Dabei handelt es sich dann um sog. „Skip-Metastasen“.

\section{Die pelvine Lymphonodektomie}

Die pelvine Lymphonodektomie für das Endometriumkarzinom umfasst folgende Gebiete:

- um die kaudale A. iliaca communis bzw. die Gefäßgabel, wo sich die A. iliaca communis in die A. iliaca externa und interna aufteilt

- das anteriore und mediale Lymphknotenfettgewebe im Bereich der A. und V. iliaca externa

- das Gewebe im Bereich der Fossa obturatoria zwischen der Beckenwand und dem Lig. umbilicale laterale, anterior des N. obturatorius (Abb. 1-3)

\section{Die paraaortale Lymphonodektomie}

Die paraaortale LNE umfasst entsprechend:

- die kranialen Anteile der A. iliaca communis

- das Gebiet der kaudalen V. cava inklusive des Areals der Aorta und der A. mesenterica inferior

- weiter kranial das interaorto-cavale Gewebe bis zum Nierenstiel, welcher durch die V. renalis sichtbar wird 
Es sei hier darauf hingewiesen, dass teilweise in der englischen und amerikanischen Literatur im Vergleich zu Deutschland die LNE etwas zurückhaltender angewandt wird.

Die potenziellen Risiken des Eingriffs liegen in der Verletzung von größeren Gefäßen und Nerven, dem Auftreten eines Lymphödems (5-38\%) mit konsekutiver Cellulitis und massiver Minderung der Lebensqualität, weswegen einige Autoren die Limitierung der pelvinen LNE auf den Bereich oberhalb der V. iliaca circumflexa unter Aussparung der kaudaleren Anteile empfehlen [12-14].

Mehrere Studien befassen sich mit der Frage, inwieweit eine LNE die Prognose der Patientin verbessert. Es gibt Hinweise, dass womöglich nur eine bestimmte Gruppe von Erkrankten mit hohem Risiko der Metastasierung von diesem Vorgehen in der Gesamtprognose profitiert.

\section{Entscheidungskriterien}

\section{Risikoklassifikation}

Die praktische Entscheidung für oder gegen eine Lymphonodektomie fällt auf Grundlage einer Risikoklassifikation, welche im Wesentlichen folgende Parameter umfasst (siehe auch oben):

- Grading

- Infiltrationstiefe

- Histologietyp

Low-Risk. Hierzu gehören endometrioide Adenokarzinome (G1 oder G2), die weniger als die Hälfte des Myometriums infiltrieren und auf das Corpus uteri beschränkt sind [10].

High-Risk. Diese Gruppe beinhaltet die Typ-II-Karzinome (serös, klarzellig, papillär oder adenosquamös), G3-Tumore, eine Infiltration > 50\% des Myometriums sowie Befunde mit Befall des Zervixstromas.

Tumore, die auf die endometriale Schleimhaut begrenzt sind oder das Myometrium weniger als $50 \%$ der gesamten Myometriumdicke infiltrieren (pT1a), haben bei einem zusätzlich vorliegenden hohen Differenzierungsgrad (G1) ein sehr geringes Risiko der pelvinen und paraaortalen LN-Metastasierung. Deswegen soll bei endometrioiden Karzinomen im Stadium pT1a und G1 oder G2 bei makroskopisch unauffälligen Lymphknoten keine LNE durchgeführt werden [15].

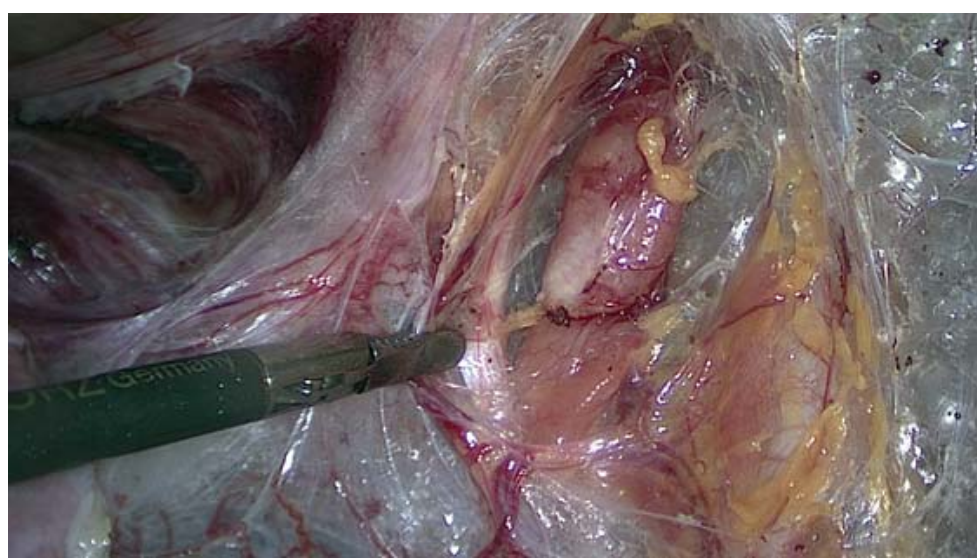

Abb. 1 Pelvine LNE iliacal extern rechts, im Bereich der Fasszange ist das Lig. umbilicale laterale sichtbar.

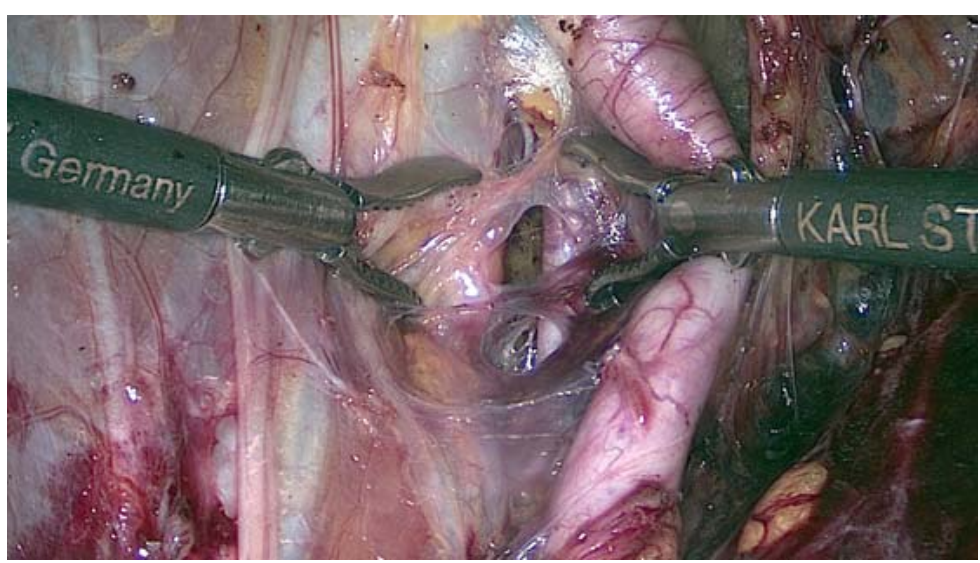

Abb. 2 Pelvine LNE links (Areal zwischen A. und V. iliaca externa).

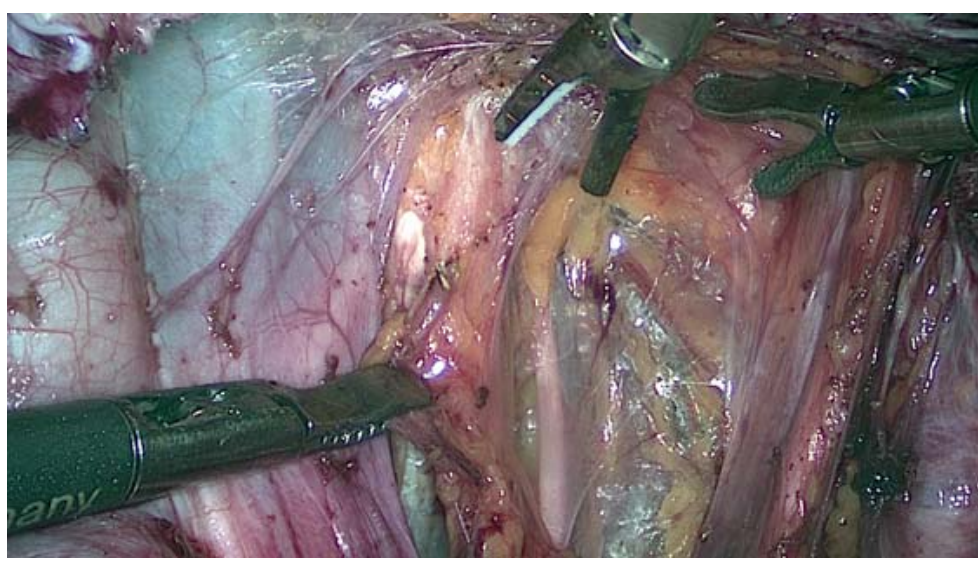

Abb. 3 Pelvine LNE links im Bereich der Fossa obturatoria, N. obturatorius (Bildmitte). 
In der Low-Risk-Konstellation werden in etwa 3-5\% der Fälle Lymphknotenmetastasen nachgewiesen [16].

Ganz im Gegensatz hierzu muss bei einer Infiltration des Myometriums über die Hälfte der Gesamtdicke (pT1b) und/oder niedriger Differenzierung (G3) in bis zu fast $40 \%$ mit LK-Metastasen gerechnet werden. Auch seröse und klarzellige Karzinome zeigen ein höheres Risiko für einen retroperitonealen LK-Befall. Als Konsequenz ergibt sich aus diesen Befunden die Notwendigkeit zur systematischen pelvinen und paraaortalen Lymphonodektomie.

\section{Tipp für die Praxis}

Besteht für das Endometriumkarzinom die Indikation zur Lymphonodektomie, so wird die pelvine und die paraaortale LNE bis zum Nierenstiel durchgeführt. Dies ist Bestandteil des operativen Stagings. Die pelvine und die paraaortale LNE können bei vorhandener Expertise und bestehendem Setting per Laparoskopie durchgeführt werden.

\section{Tipp für die Praxis}

Die Infiltrationstiefe, welche bei einem endometrioiden G1- oder G2-Karzinom über die LNE entscheidet, lässt sich nur am Hysterektomiepräparat zuverlässig feststellen. Wenn möglich, sollte diese im Rahmen einer Schnellschnittuntersuchung diagnostiziert werden, dann kann in gleicher Sitzung die Entscheidung zur LNE getroffen werden.

\section{Rationale des Sentinel-Konzepts}

Kontrovers sind Publikationen, die einen Überlebensvorteil nach pelviner und paraaortaler LNE belegen [18], oder diesen aber nicht eindeutig nachweisen können [19].

Da das LK-Staging eine wichtige Rolle in der Identifizierung von Patientinnen spielt, die eine adjuvante Therapie erhalten, ist es bedeutsam, diejenigen LK zu detektieren, die mit großer Wahrscheinlichkeit betroffen sind [20]. In einer retrospektiven Multicenterstudie konnte gezeigt werden, dass 16\% der Patientinnen mit Endometriumkarzinom aus der niedrigen oder mittleren Risikogruppe positive Sentinel-LK aufwiesen [21].

Bislang bildeten meistens kleine Fallserien, bei denen Technetium-99m oder Blaulösung eingesetzt wurden, die Datengrundlage. Die Injektion dieser Substanzen ist prinzipiell an folgenden Lokalisationen möglich [22]:

- perizervikal

- hysteroskopisch peritumoral

- fundal peritumoral

Die perizervikale Injektion war in einer Metaanalyse mit einer höheren Rate an detektierten Sentinel-LK verbunden, wohingegen der hysteroskopische und der subseröse Ansatz mit geringeren Ergebnissen hinsichtlich Detektionsrate und Sensitivität vergesellschaftet waren, wenn sie nicht mit anderen Injektionstechniken kombiniert wurden [25]. Daraus leitet sich die Empfehlung ab, dass eine duale, kombinierte perizervikale Applikation von Technetium-99m und Blaulösung erfolgen sollte $[22,26]$.

\section{Ausblick}

Die Sentinel-LNE ist trotz vielversprechender Ergebnisse noch nicht als Standard anzusehen und soll daher Zentren im Rahmen von interdisziplinären Studien vorbehalten sein. Es lässt sich aber erwarten, dass das Konzept das operative Staging in der Zukunft v.a. für die Low-Risk-Karzinome beeinflussen wird und die Morbidität einer systematischen pelvinen und paraaortalen Lymphonodektomie in dieser Patientinnengruppe senken kann. 


\section{Omentektomie}

Liegt histologisch ein Typ-II-Karzinom vor (seröse oder klarzellige Komponenten) oder es handelt sich um ein endometrioides Korpuskarzinom, bei dem intraoperativ ein Serosa- oder Adnexbefall ersichtlich ist (T3a), wird der Tumor analog des Ovarialkarzinoms therapiert: Neben dem o.g. Standardvorgehen müssen auch zusätzlich die Omentektomie und die Entnahme multipler Peritonealbiopsien erfolgen.

Während also eine routinemäßige Omentektomie selten zu einem therapierelevanten Up-Staging führt und daher beim endometrioiden Korpuskarzinom nicht notwendig ist, solange keine Metastasen bei der intraoperativen Inspektion vorliegen [27], spielt die Omentektomie für das exakte Staging bei nicht endometrioiden Tumoren sowie bei Adnexmetastasen und peritonealer Disseminierung eine signifikante Rolle [28].

\section{Therapie in den fortgeschrittenen Stadien II-IV}

\section{Stadium II}

Entsprechend der aktuellen Empfehlungen sollte im Stadium II (Befall des Zervixstromas, aber keine Ausbreitung über den Uterus hinaus) keine radikale Hysterektomie durchgeführt werden [15].

Hier fehlen ausreichend prospektiv randomisierte Studien, eine retrospektive Analyse von Takano et al. fand, dass die Art der Hysterektomie (einfach versus radikal) kein Prognosefaktor bei Patientinnen mit Zervixbefall war und perioperative Komplikationen sowie Nebenwirkungen im weiteren Verlauf (Blasenfunktionsstörungen) in der radikal hysterektomierten Gruppe häufiger waren [29].

Kasuistik zum fallorientierten Lernen

\section{Symptome und Klinik}

68-jährige postmenopausale Patientin mit seit ca. 3 Monaten unregelmäßigen unterregelstarken Blutungen.

\section{Anamnese}

Unregelmäßige Vorsorge. Nebenerkrankungen, Hypertonie, Adipositas, Diabetes, gering sensible Polyneuropathie, axiale Ösophagushernie, Allergien gegen Penicillin, Chloramphenicol, Jod. Z. n. 2 × Längslaparotomie bei Sigmadivertikulitis, keine HRT.

Familienanamnese: Die Mutter und Großmutter hätten beide Darmkrebs gehabt, letztere sei relativ jung verstorben.

\section{Verdachtsdiagnose}

Endometriumkarzinom

\section{Untersuchung und Diagnostik}

Abdomen adipös, weich, reizlose, aber verbreiterte Längslaparotomienarbe, äußeres Genitale o. B. Spekulum: unauffällig, etwas Blutung ex CK. Rektovaginal, soweit bei Adipositas beurteilbar, Uterus normal groß, fraglich etwas nach links fixiert, linker Adnexbereich mit teigiger Resistenz, rechts unauffällig.
Transvaginaler Ultraschall: Uterus nach links fixiert, leicht vergrößert, Endometrium deutlich verdickt, ca. $12 \mathrm{~mm}$, etwas irregulär begrenzt, beide Adnexbereiche unauffällig. Aufgrund der Fixierung des Uterus werden Adhäsionen im linken Unterbauch erwartet.

\section{Weiterführende Diagnostik}

Histologische Abklärung mittels Hysteroskopie und fraktionierter Abrasio.

Zervixabradat makroskopisch und histologisch unauffällig. Korpusabradat mit viel Gewebe, makroskopisch suspekt. Histologisch zeigen sich viele Anteile eines gering differenzierten endometrioiden Adenokarzinoms, G3. In der immunhistochemischen Untersuchung zeigen die Tumorzellen eine Expression von MLH1, PMS2, MSH2 und MSH6. Somit liegt hier ein mikrosatellitenstabiler Tumor vor. Das spricht dafür, dass es sich um kein HNPCC-assoziiertes, sondern ein sporadisches metachrones Karzinom des Endometriums handelt.

\section{Therapie}

Es besteht ein Endometriumkarzinom der High-Risk-Gruppe (G3). Daher Planung der Hysterektomie, Adnexektomie, DouglasSpülzytologie, systematische pelvine und paraaortale LNE. Auf Wunsch der Patientin auch im Z. n. nach Längslaparotomie Versuch eines minimalinvasiven Vorgehens per LSK. Zunächst Durchführung der LSK über Palmer'schen Punkt: es zeigen sich im Z.n. Darmvoroperation massive Verwachsungen im Mittel-/Unterbauch, v. a. links. Der Adnexbereich links und der Uterus sind mit dem Rektosigmoid an der linken Beckenwand postoperativ durch Adhäsionen fixiert. Aufgrund des „Frozen Pelvis“ Entscheidung zur Konversion auf Re-Längslaparotomie mit Hysterektomie, Adnexektomie, Zytologie und pelvine und paraaortale Lymphonodektomie nach ausgedehnter Adhäsiolyse und Ureterolyse.

Histologie: endometrioides Adenokarzinom, pT1b (16 mm), pN0 (0/44, pelvin links: 16, rechts: 18 , paraaortal 10$)$. Unauffälliger postoperativer Verlauf, im Intervall Bestrahlung.

\section{Nachsorge}

Gemäß den Empfehlungen der Interdisziplinären Leitlinie der Deutschen Krebsgesellschaft e. V. (DKG) und der Deutschen Gesellschaft für Gynäkologie und Geburtshilfe (DGGG). 


\section{Stadium III}

Das Ziel der Operation im fortgeschrittenen Stadium (Stadium III: intraperitoneal, Vagina- oder Parametrienbefall, Metastasen in Becken- und/oder paraaortalen LK) ist möglichst die Entfernung von sichtbaren Tumoranteilen im Sinne einer Zytoreduktion zur Verbesserung der weiteren adjuvanten Maßnahmen $[15,30]$. Unter Umständen wird also zusätzlich zu den oben bereits genannten Schritten (Hysterektomie, Adnexektomie, Lymphonodektomie, Omentektomie) auch die partielle oder komplette Kolpektomie erforderlich.

Letztere kann vermieden werden, wenn es gelingt, vaginale Tumoranteile zu exzidieren und dann eine postoperative Kontaktbestrahlung anzuschließen.

\section{Stadium IV}

Zunächst ist in diesem Stadium (Blasen- und Rektumbefall) eine richtige Diagnosestellung erforderlich. Es wird daher eine Narkoseuntersuchung mit Entnahme von Gewebeproben empfohlen, die in gleicher Sitzung von einer Zystoskopie und Rektoskopie begleitet wird. Die nächste relevante Frage betrifft die Fernmetastasierung, sodass sich ein Ganzkörperstaging mit CT anschließen sollte. Die weitere Therapie erfolgt individualisiert unter Berücksichtigung des Patientenwunsches und der Komorbiditäten. Eine vordere oder hintere Exenteration kann bei isoliertem Befall hauptsächlich zur Tumorkontrolle sinnvoll sein und dient dann der Vermeidung von Kloakenbildung und Darmobstruktion [30,31]. Diese Operationen haben einen sehr hohen Schwierigkeitsgrad und sollten daher an speziellen Zentren mit der Möglichkeit der interdisziplinären Operation (Allgemeinchirurgie, Urologie, Gefäßchirurgie) geplant und ausgeführt werden.

\section{Therapie von Rezidiv und Metastasen}

Basierend auf den derzeit gültigen Empfehlungen sollten resezierbare Befunde primär komplett operativ entfernt werden, andernfalls wird die Strahlentherapie eingesetzt. Wenn weder Operation noch Bestrahlung möglich sind, ist die palliative Systemtherapie zu erwägen [15].

\section{Tipp für die Praxis}

Bei fortgeschrittenen Stadien ist die Therapie vorwiegend auf die Entfernung sichtbarer Tumoranteile und die lokale Kontrolle zentriert. Als Modalitäten kommen hierfür zum Einsatz:

- Operation (u.U. Kolpektomie, Exenteration)

- Radiatio (Teletherapie, Brachytherapie)

- Gestagene (bei östrogen- und/oder progesteronrezeptorpositiven Karzinomen)

- Chemotherapie (Kombination gegenüber der Monotherapie bez. Gesamtüberleben nur minimal im Vorteil)

\section{Kernaussagen}

Das Endometriumkarzinom ist der häufigste maligne Tumor des inneren Genitales. Das Hauptsymptom besteht in der vaginalen Blutung, $v$. a. in der Postmenopause. Diese muss durch Hysteroskopie und fraktionierte Abrasio abgeklärt werden. Die Standardtherapie besteht in der Hysterektomie, der bilateralen Adnexektomie und der Peritonealspülzytologie, welche mittlerweile meistens per laparoscopiam möglich ist. Je nach zusätzlichen Risikokriterien ist ein operatives Staging mit Erweiterung des Vorgehens notwendig, welches die systematische LNE und die Omentektomie umfassen kann. Die Sentinel-LNE wird voraussichtlich das Staging bei Low-Risk-Fällen modifizieren, ist aber aktuell noch Studien vorbehalten.

Ähnlich wie bei anderen Tumorentitäten wird voraussichtlich in naher Zukunft auch die molekulare Klassifikation der verschiedenen Typen des Endometriumkarzinoms für die Therapiestrategie eine vielversprechende Rolle spielen [32]. 


\section{Über die Autoren}

\section{Bernhard Krämer}

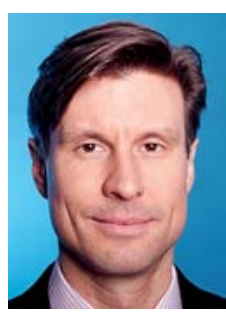

Priv.-Doz. Dr. med. Jahrgang 1974. Studium der Humanmedizin in Greifswald, Kiel, Sheffield (England) und Ulm. Promotion zum Dr. med. am Institut für Toxikologie der Universität Kiel. 2007 Facharzt für Gynäkologie und Geburtshilfe. Seit 2012 Ltd. Oberarzt an der Universitätsfrauenklinik Tübingen. April 2014 Habilitation und Venia Legendi für das Fach Frauenheilkunde und Geburtshilfe, Eberhard-Karls-Universität Tübingen. Aktuelle wissenschaftliche Schwerpunkte: gynäkologische Onkologie, experimentelle und minimalinvasive Operationstechniken, Ursachen für die Adhäsionsbildung im kleinen Becken und deren Prävention.

\section{Florin-Andrei Taran}

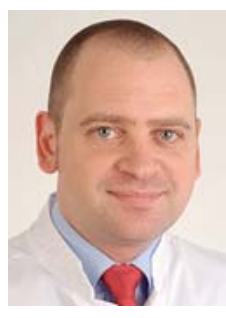

Prof. Dr. med. Jahrgang 1978. Studium der Humanmedizin an der Universität Heidelberg. Promotion an der Abteilung für Angewandte Tumorbiologie, Institut für Pathologie der Universität Heidelberg 2007. 2005-2010 wissenschaftlicher Mitarbeiter der Universitätsfrauenklinik Magdeburg. Seit Juli 2010 wissenschaftlicher Mitarbeiter der Universitätsfrauenklinik Tübingen. März 2011 Facharztanerkennung Frauenheilkunde und Geburtshilfe. Mai 2012 Oberarzt und Leiter der Arbeitsgruppe Tumorzelldissemination. Oktober 2012 Habilitation und Venia legendi für das Fach Frauenheilkunde und Geburtshilfe, Eberhard-Karls-Universität Tübingen. Juni 2015 außerplanmäßige Professur Eberhard-Karls-Universität Tübingen. Wissenschaftliche Schwerpunkte: Tumorzelldissemination Leiomyomvarianten, minimalinvasive Operationstechniken.

\section{Sara Y. Brucker}

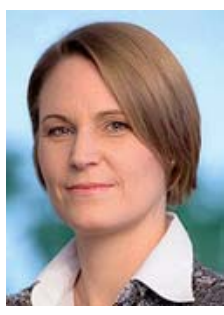

Prof. Dr. med. Jahrgang 1974. Studium der Humanmedizin an der AlbertLudwigs-Universität Freiburg. 2001 Assistenzärztin an der Universitätsfrauenklinik Tübingen. 2005 Facharztanerkennung, 2009 Habilitation und Venia Legendi. Seit 2010 Leiterin des Zentrums für seltene genitale Fehlbildungen im Zentrum für seltene Erkrankungen. 2011 Stellvertretende Ärztliche Direktorin und Professur für Spezielle Gynäkologie und Frauenheilkunde an der Universitätsfrauenklinik Tübingen. Seit 2014 Ärztliche Direktorin des Forschungsinstituts für Frauengesundheit und Stellvertretende GF Ärztliche Direktorin des Departments für Frauengesundheit an der Universität Tübingen.

\section{Interessenkonflikt}

Für die Autoren besteht in Bezug auf das Manuskript kein Interessenkonflikt.

\section{Korrespondenzadresse}

PD Dr. Bernhard Krämer

Universitätsfrauenklinik Tübingen

Calwerstr. 7

72076 Tübingen

E-Mail: bernhard.kraemer@med.uni-tuebingen.de 


\section{Literatur}

1 Setiawan VW, Yang HP, Pike MC et al. Type I and II endometrial cancers: have they different risk factors? J Clin Oncol 2013; 31 : 2607-2618

2 Brinton LA, Felix AS, McMeekin DS et al. Etiologic heterogeneity in endometrial cancer: evidence from a Gynecologic Oncology Group trial. Gynecol Oncol 2013; 129: 277-284

3 Levine DA, Lin O, Barakat RR et al. Risk of endometrial carcinoma associated with BRCA mutation. Gynecol Oncol 2001; 80: 395-398

4 Segev Y, Iqbal J, Lubinski J et al. The incidence of endometrial cancer in women with BRCA1 and BRCA2 mutations: an international prospective cohort study. Gynecol Oncol 2013; 130: $127-131$

5 Berek J, Adashi E, Hillard P. Novak's Gynecology. 12th ed. Baltimore: Lippincott, Williams \& Wilkins; 1996

6 Lahti E, Blanco G, Kauppila A et al. Endometrial changes in postmenopausal breast cancer patients receiving tamoxifen. Obstet Gynecol 1993; 81: 660-664

7 Palomba S, Falbo A, Mocciaro R et al. Laparoscopic treatment for endometrial cancer: a meta-analysis of randomized controlled trials (RCTs). Gynecol Oncol 2009; 112: 415-421

8 Berretta R, Merisio C, Melpignano M et al. Vaginal versus abdominal hysterectomy in endometrial cancer: a retrospective study in a selective population. Int J Gynecol Cancer 2008; 18: 797-802

9 Susini T, Massi G, Amunni G et al. Vaginal hysterectomy and abdominal hysterectomy for treatment of endometrial cancer in the elderly. Gynecol Oncol 2005; 96: 362-367

10 Mariani A, Dowdy SC, Cliby WA et al. Prospective assessment of lymphatic dissemination in endometrial cancer: a paradigm shift in surgical staging. Gynecol Oncol 2008; 109: 11-18

11 Abu-Rustum NR, Gomez JD, Alektiar KM et al. The incidence of isolated paraaortic nodal metastasis in surgically staged endometrial cancer patients with negative pelvic lymph nodes. Gynecol Oncol 2009; 115: 236-238

12 Todo Y, Yamamoto R, Minobe S et al. Risk factors for postoperative lower-extremity lymphedema in endometrial cancer survivors who had treatment including lymphadenectomy. Gynecol Oncol 2010; 119: 60-64

13 Abu-Rustum NR, Alektiar K, lasonos A et al. The incidence of symptomatic lower-extremity lymphedema following treatment of uterine corpus malignancies: a 12-year experience at Memorial Sloan-Kettering Cancer Center. Gynecol Oncol 2006; 103: 714-718

14 Burke WM, Orr J, Leitao M et al.; SGO Clinical Practice Endometrial Cancer Working Group. Endometrial cancer: a review and current management strategies: part I. Gynecol Oncol 2014; 134: $385-392$

15 Arbeitsgemeinschaft Gynäkologische Onkologie. Empfehlungen für die Diagnostik und Therapie des Endometriumkarzinoms. Aktualisierte Empfehlungen der Kommission Uterus auf Grundlage der S2 k Leitlinie (Version 1.0, 1.6. 2008) ohne Angabe der Evidenzlevel und Empfehlungsgrade. Herausgegeben von der Kommission Uterus der Arbeitsgemeinschaft Gynäkologische Onkologie e. V., April 2013. Im Internet: http://www.ago-online. de/fileadmin/downloads/leitlinien/uterus/empfehlungen_diagnostik_therapie_EC.pdf; Stand: 29.01.2016
16 Watanabe M, Aoki Y, Kase $\mathrm{H}$ et al. Low risk endometrial cancer: a study of pelvic lymph node metastasis. Int J Gynecol Cancer 2003; 13: 38-41

17 Burke TW, Levenback C, Tornos C et al. Intraabdominal lymphatic mapping to direct selective pelvic and paraaortic lymphadenectomy in women with high-risk endometrial cancer: results of a pilot study. Gynecol Oncol 1996; 62: 169-173

18 Todo Y, Kato H, Kaneuchi M et al. Survival effect of para-aortic lymphadenectomy in endometrial cancer (SEPAL study): a retrospective cohort analysis. Lancet 2010; 375: 1165-1172

19 ASTEC study group, Kitchener H, Swart AM, Qian Q et al. Efficacy of systematic pelvic lymphadenectomy in endometrial cancer (MRC ASTEC trial): a randomised study. Lancet 2009; 373: $125-136$

20 Abu-Rustum NR. The increasing credibility of sentinel lymph node mapping in endometrial cancer. Ann Surg Oncol 2013; 20: 353-354

21 Ballester M, Naoura I, Chéreau E et al. Sentinel node biopsy upstages patients with presumed low- and intermediate-risk endometrial cancer: results of a multicenter study. Ann Surg Oncol 2013; 20: $407-412$

22 Brucker S, Taran FA, Wallwiener D et al. Sentinel lymph node mapping in endometrial cancer: a concept ready for clinical routine? Arch Gynecol Obstet 2014; 290: 9-11

$23 \mathrm{Kim} \mathrm{CH}$, Soslow RA, Park KJ et al. Pathologic ultrastaging improves micrometastasis detection in sentinel lymph nodes during endometrial cancer staging. Int J Gynecol Cancer 2013; 23: 964-970

$24 \mathrm{Kim} \mathrm{CH}$, Khoury-Collado F, Barber EL et al. Sentinel lymph node mapping with pathologic ultrastaging: a valuable tool for assessing nodal metastasis in low-grade endometrial cancer with superficial myoinvasion. Gynecol Oncol 2013; 131: 714-719

25 Kang S, Yoo HJ, Hwang JH et al. Sentinel lymph node biopsy in endometrial cancer: meta-analysis of 26 studies. Gynecol Oncol 2011; 123: $522-527$

26 Ballester M, Dubernard G, Lécuru F et al. Detection rate and diagnostic accuracy of sentinel-node biopsy in early stage endometrial cancer: a prospective multicentre study (SENTI-ENDO). Lancet Oncol 2011; 12: 469-476

27 Ozdal B, Unlu BS, Yalcin HR et al. Role of omentectomy and appendectomy in surgical staging of endometrioid endometrial cancer. Eur J Gynaecol Oncol 2013; 34: 322-324

28 Sakai K, Yamagami W, Susumu N et al. Pathological factors associated with omental metastases in endometrial cancer. Eur J Gynaecol Oncol 2015; 36: 397-401

29 Takano M, Ochi H, Takei Y et al. Surgery for endometrial cancers with suspected cervical involvement: is radical hysterectomy needed (a GOTIC study)? Br J Cancer 2013; 109: 1760-1765

30 Wallwiener D, Jonat W, Kreienberg R, Hrsg. Atlas der gynäkologischen Operationen. 7. Aufl. Stuttgart: Thieme; 2008

31 Fehm T, Taran FA, Becker S. Die operative Therapie des Endometriumkarzinoms. Frauenheilkunde up2date 2010; 4: 223-233

32 Rutgers JK. Update on pathology, staging and molecular pathology of endometrial (uterine corpus) adenocarcinoma. Future Oncol 2015; 11: $3207-3218$ 


\section{CME-Fragen}

\section{CME.thieme.de}

cME-Teilnahme

- Viel Erfolg bei lhrer CME-Teilnahme unter http://cme.thieme.de

- Diese Fortbildungseinheit ist 12 Monate online für eine CME-Teilnahme verfügbar.

- Sollten Sie Fragen zur Online-Teilnahme haben, unter

http://cme.thieme.de/hilfe finden Sie eine ausführliche Anleitung.

\section{$1 \quad$ Was trifft für das sog. Lynch-Syndrom zu?}

A Es handelt sich um eine autosomal-rezessiv vererbte Mutation.

B Untypisch ist eine Mikrosatelliteninstabilität.

C Gehäuft kommen Endometriumkarzinome mit jüngerem Erkrankungsalter vor.

D Das Auftreten kolorektaler Karzinome ist selten.

E Der Nachweis erfolgt durch Transvaginalsonografie.

2 Welche Aussage zu den Risikofaktoren trifft zu?
A Tamoxifen-Einnahme hat eine Schutzwirkung auf das Endometrium.
B Nulliparität vermindert das Risiko.
C Adipositas ist ein typischer Risikofaktor.
D Das Risiko nimmt mit höherem Lebensalter ab.
E Diabetes ist kein Risikofaktor.

\section{Welche Aussage trifft zu?}

A Die Endometriumhyperplasie ist die typische Vorstufe für ein Typ-II-Endometriumkarzinom.

B Bei der atypischen Endometriumhyperplasie kann unter bestimmten Voraussetzungen ein konservatives Management erfolgen.

C Bei der atypischen Endometriumhyperplasie muss im Rahmen der operativen Therapie eine Lymphonodektomie erfolgen.

D Hyperplasien ohne Atypie haben kein Entartungsrisiko.

E Eine konservative Hormontherapie bei der Endometriumhyperplasie erfolgt mit Östrogenen.

4 Welche Aussage trifft zur Standardtherapie des Endometriumkarzinoms nicht zu?
A Hysterektomie
B Adenxektomie
C Spülzytologie
D Omentektomie
E Laparotomie und Laparoskopie sind mögliche Zugangswege.

5 Welche Aussage zur Lymphonodektomie (LNE) trifft zu?
A Wenn die pelvine LNE durchgeführt wurde, ist die paraaortale LNE niemals erforderlich.
B Die pelvine LNE ist durch Entfernung von Fettlymphknotengewebe im Bereich der A. mesenterica inferior gekennzeichnet.
C Die LNE muss stets per Laparotomie erfolgen.
D Die paraaortale LNE ist per Laparoskopie nicht möglich.
E Sog. „Skip-Metastasen“ bezeichnen positive paraaortale LK ohne das Vorliegen von pelvinen Metastasen. 


\section{Was gilt für die Risikoklassifizierung?}

A Seröse Karzinome gehören zur Gruppe des höheren Risikos.

B Die Infiltrationstiefe spielt bei der Einteilung keine Rolle.

C Sie muss bei der Entscheidung für oder gegen eine Lymphonodektomie nicht beachtet werden.

D In der Low-Risk-Gruppe genügt die paraaortale LNE.

E Das Grading wird nicht herangezogen.

\section{Welche Aussage trifft nicht zu?}

A Die Sentinel-LNE basiert auf einer Technetium-Injektion perizervikal.

B Eine Technetium-Injektion sollte auch mit einer präoperativen Injektion von Blaufarbstoff kombiniert werden.

C Das Sentinel-Konzept zielt v. a. auf die Gruppe der Low-Risk-Karzinome.

D Das Sentinel-Konzept läuft unter Studienbedingungen.

E Das Sentinel-Konzept kann mittlerweile als Standard betrachtet werden.

8 Welche Aussage trifft für das Endometriumkarzinom mit Zervixstromabefall zu?
A Im Stadium II sollte keine radikale Hysterektomie erfolgen.
B Es besteht die typische Indikation für eine Parametrienresektion.
C Es handelt sich um einen Low-Risk-Fall.
D Die Omentektomie ist typischer Bestandteil der operativen Therapie.
E Es kann immer durch die Hysteroskopie diagnostiziert werden.

\section{9}

\section{Welche Aussage trifft zu?}

A Im Stadium pT1a, G1 ist die systematische LNE fakultativ.

B Im Stadium pT1a, G1 soll keine LNE durchgeführt werden.

C Alle Endometriumkarzinome müssen adjuvant bestrahlt werden.

D Eine Kolpektomie ist bei Endometriumkarzinom nie eine Therapieoption.

E Bei Rezidiv und Metastasen eines Endometriumkarzinoms sollten keine Gestagene gegeben werden.

\section{Welche Aussage trifft zu?}

A Typischerweise erkrankt die prämenopausale Patientin an einem Endometriumkarzinom.

B Das Endometriumkarzinom ist typischerweise BRCA-assoziiert.

C Die meisten Patientinnen erkranken um das 50. Lebensjahr.

D Die Inzidenz der Erkrankung nimmt mit steigendem Lebensalter zu.

E Das Endometriumkarzinom ist häufiger als das Mammakarzinom. 